in full in Chapter 1 of the Code of Practice, will be referred to throughout the Code. We believe that this enhanced status, together with the embedding of the training materials in a strongly values-based approach, provides an opportunity to move mental health practice in compulsion firmly towards the recovery-based approach advocated by Roberts and his co-authors.

\section{Declaration of interest}

K.W.M.F and M.K. are both involved in developing the training materials to support the Mental Health
Act 2007, M.K. as National Training Lead on the Implementation of the Care Services Improvement Partnership, and K.W.M.F as Special Adviser for Values-Based Practice at the Department of Health.

\section{References}

Fulford, K. W. M. (1989) Moral Theory and Medical Practice. Cambridge University Press.

Roberts, G., Dorkins, E., Wooldridge, J. \& Hewis, E. (2008) Detained - what's my choice? Part 1: Discussion. Advances in Psychiatric Treatment, 12, 172-180.

Woodbridge, K. \& Fulford, K. W. M. (2004) Whose Values? A Workbook for Values-Based Practice in Mental Health Care. Sainsbury Centre for Mental Health.

\title{
Detained - what's my choice? Part 2: Conclusions and recommendations ${ }^{\dagger}$
}

\author{
Eluned Dorkins, Glenn Roberts, James Wooldridge \\ \& Elaine Hewis
}

\begin{abstract}
We have developed this succession of articles as a series of iterative steps, each seeking to uphold the recovery values of co-working and collaboration, looking for agreement and commonality but valuing equally diverse viewpoints and difference. Our conclusion is that this is the beginning of a creative dialogue on choice as a route to recovery for people who are psychiatrically detained. We commend our method of engaging with the inevitable tensions and dilemmas by: clarifying the story behind difficult interactions, identifying the relevant guiding principles and jointly working to explore from different viewpoints what can be done to promote recovery.
\end{abstract}

There can sometimes seem to be unbridgeable chasms of perspective between professionals and detained patients. Our aim was to examine some of these differences and talk together about how best to work in service of the common aim of recovery.

${ }^{+}$To be read in conjunction with pp. 172-180, 181-182 and $183-184$, this issue.
We are grateful to our commentators for putting our observations and viewpoints (Roberts et al, 2008, this issue) in a broader context. Fulford \& King (2008, this issue) offer an intriguing glimpse into future guidance on working with people subject to compulsory treatment. They illustrate a close parallel to our own method of working to clarify guiding principles through which values can be applied to specific situations.

Eluned Dorkins is a consultant forensic psychiatrist at Langdon Hospital, Dawlish, Devon. Glenn Roberts is a consultant in rehabilitation and recovery in Devon (Wonford House Hospital, Dryden Road, Exeter EX2 5AF, UK. Email: glennroberts@doctors.org.uk). James Wooldridge is a service avoider, freelance trainer/consultant/speaker and regional coordinator for the Open Up project. Elaine Hewis is a survivor and carer, consultant trainer and lecturer in mental health issues. 


\section{Box 1 Resources}

Choice

Care Services Improvement Partnership's (CSIP's) Choices in Mental Health programme (www.mhchoice.csip.org.uk)

\section{Recovery}

www.recoverydevon.co.uk

Peer support

www.mentalhealthpeers.com

\section{Self-management}

The Wellness Recovery Action Plan (WRAP) (www.mentalhealthrecovery.com)

Implementing the Mental Health Act 2007

http:/ / mhact.csip.org.uk

In terms of bottom-up and top-down approaches, we may characterise ours as an attempt to meet in the middle. Copeland \& Mead (2008, this issue) draw on their many years of working for user empowerment, effective self-management and advocacy to value our efforts as a beginning, but only a beginning, and there is need for a continuing dialogue through which progressive, humane and recovery-oriented approaches may be developed with people in great difficulties. This has implications for how both practitioners and people who use services take responsibility for working for recovery outcomes in future. Given the inequalities of power that characterise working with detained patients, they remind us to focus on the needs of the person being served. All mental healthcare professionals want to put patients first (Box 1) but how they propose to do it may vary with the underlying assumptions and values that colour their judgements about how this is to be done. We share the hope that even in these circumstances there is scope for greater negotiation and collaboration, choice and its attendant responsibilities. It would appear that the training materials being prepared by Fulford \& King may be a means of exploring that end.

\section{The value and values of collaboration}

We began with a question, which we sought to answer by engaging in a search for how choice could be activated as a support for recovery for people who are detained in psychiatric services. But we also consider our method and the model of co-working that we developed through this project to be as much of a result as our specific findings and refined viewpoints. All four of us have greatly valued our collaboration and gained much from working in partnership. J.W. and E.H. have also valued our reflective process as part of their on-going journey in personal recovery, through which they have reappraised some of their experiences while detained. Although we have met 'after the event', we suggest that our method of clarifying the story surrounding difficult interactions, working out the salient guiding principles and jointly considering what can be done to promote recovery from differing viewpoints could be a process that finds application 'in the moment' in clinical settings as well. And there is additional value in the reflective support of expert colleagues and advocates.

In reviewing our viewpoints, three familiar themes stand out: the issue of power and the need for trust; the issue of rules and the need for clear thinking based on explicit and fair principles; and the issue of communication and the need for mutual respect that fosters the ability to listen and learn from one another.

\section{Limitations and strengths}

During the process of working on this article and with the benefit of 20/20 hindsight we all agreed that detention, when needed, can be a route to recovery. There are many limitations associated with a subjective and reflective article from a small sample of opinions. A specific restriction may be that we have sought and focused on common ground, which may have limited how much of the rich diversity of opinion and experience we were able to capture in our collaboration. However, it would be difficult to capture here the full range of complex interactions, different settings and services that look after detained patients. We also acknowledge that other practitioners and service users may offer different views. We offer this co-authored article as much for upholding the value of an experience of collaborative working as for the information it contains. We see this project as illustrative of Valsraj \& Gardner's (2007) concluding proposition that embracing choice involves 'moving on from a paternalistic and authoritarian approach to one that champions partnership and dialogue'. But we also believe that at the 'deep end' of psychiatry, where there are major concerns for personal and public safety, there remains a need for boundary and what may be regarded as compassionate paternalism, without which healthcare professionals risk neglecting their responsibility to people at times when they are so overwhelmed by their problems that they lack capacity to be fully responsible for themselves.

This is a complex matter. It will always be difficult to know how best to act at the interface where there is a 'direct conflict of values' (Fulford \& King, 2008, this issue), whether morbidly inspired or not. Resolution 
of these tensions and responsible decision-making do need complex and careful debate. They also require considerable self-awareness in those involved and often the reflective opportunities provided by peers or supervisors.

\section{In search of balance}

There are many contributing processes that help or hinder recovery for detained patients, and the judicious exercise of choice appears to be a key consideration. We think that the issue is about optimising rather than maximising choice in ways that reflect an individual's capacity both to make good choices and to responsibly take constructive risks. There is an inevitable need to balance choice with contextual constraints (legal, social, financial and clinical), which will differ in forensic and open settings. The task is to search for the difficult and shifting balance between too much and too little control, over- and under-identification with the patient's current experience, and too tight or too loose a use of boundaries, all in search for a way of working that supports both safety and progress - recovery.

As patient choice becomes a service and commissioning priority, there is a need for sensitivity and wisdom to avoid naive and simplistic interpretations in search of this shifting balance - a balance that optimally supports progress in recovery alongside considerations of safety for both individuals and society.

We suggest that engaging in collaborative discussions and explorations such as this is a contribution in the right direction.

\section{Acknowledgement}

We gratefully acknowledge the helpful contribution of Emma Laughton to this project in the early stages.

\section{Declaration of interest}

None.

\section{References}

Copeland, M. E. \& Mead, S. (2008) Continuing the dialogue: moving towards shared risk. Invited commentary on: Detained - what's my choice. Part 1. Advances in Psychiatric Treatment, 14, 181-182.

Fulford, K. W. M. \& King, M. (2008) A values-based perspective on good practice in compulsion. Invited commentary on: Detained - what's my choice. Part 1. Advances in Psychiatric Treatment, 14, 183-186.

Roberts, G., Dorkins, E., Wooldridge, J. \& Hewis, E. (2008) Detained - what's my choice? Part 1: Discussion. Advances in Psychiatric Treatment, 12, 172-186.

Valsraj, K. M. \& Gardner, N. (2007) Choice in mental health: myths and possibilities. Advances in Psychiatric Treatment, 13, 60-67.

\section{MCQs}

1 Being able to offer choice:

a always depends on having more resources

b applies only to informal (voluntary) patients, not to those detained under the Mental Health Act 1983

c can be as much about the process as the outcome

d is not a goal of NHS modernisation

e has nothing to do with recovery-based practice.

2 Allowing patient choice:

a can be therapeutic in all circumstances

b can be countertherapeutic in all circumstances

c should not have to be judged in the light of contextual factors

d should, where possible, be explained to the patient and based on a reasoned rationale

e is an absolute right.

3 Participation in meaningful occupation and activity:

a is not associated with improved clinical outcomes

b does not come under the remit of treatment as part of the grounds for detention under the Mental Health Act 1983

c is not valued by service users

d should not be used as a component by which patients' progress is assessed

e should be a goal based on a team's shared understanding of its part in the overall treatment plan.

4 Living in residential settings:

a offers unlimited options for choice

b does not require the shared understanding of residents about how they can behave

c poses no challenge to staff to retain the sense of the individual

d can be countertherapeutic unless the underlying values, goals and aims of treatment are clear to counter the risk of 'care' becoming abusive

e is easy for patients and staff.

5 The concept of risk in decisions about treatment:

a is value free

b always infers a negative

c could be seen as a creative opportunity to provide new experiences in treatment planning

$\mathrm{d}$ is not influenced by wider societal norms and expectations

e need not be grounded in reasoned arguments in daily clinical practice to be transparent and defensible.

\begin{tabular}{|lllllll|}
\hline MCQ answers & & & & \\
1 & & 2 & 3 & 4 & 5 \\
a F & a F & a F & a F & a F \\
b F & b F & b F & b F & b F \\
c T & c F & c F & c F & c T \\
d F & d T & d F & d T & d F \\
e F & e F & e T & e F & e F
\end{tabular}

